\title{
Analysis of Closure Characteristics of a MEMS Omnidirectional Inertial Switch under Shock Loads
}

\author{
Yun Cao, Zhanwen Xi, Jiong Wang, and Weirong Nie \\ School of Mechanical Engineering, Nanjing University of Science and Technology, Nanjing 210094, China \\ Correspondence should be addressed to Zhanwen Xi; 4317045xi@sina.com
}

Received 1 December 2014; Accepted 16 March 2015

Academic Editor: Laurent Mevel

Copyright (C) 2015 Yun Cao et al. This is an open access article distributed under the Creative Commons Attribution License, which permits unrestricted use, distribution, and reproduction in any medium, provided the original work is properly cited.

\begin{abstract}
A preliminary theoretical method for calculating contact time of a dual mass-spring system applied to shock acceleration was proposed based on the MEMS omnidirectional inertial switch. The influence of relevant parameters on the contact time was analyzed, and the theoretical results were in agreement with the simulation predictions. The theoretical method could provide the design of MEMS inertial switch for prolonged contact time. The system stiffness of the mass-spring system in all directions was obtained by using the FE method. Dynamic contact simulation results of contact time in typical directions under the applied shock acceleration indicate that the switch has a contact time within the range of $33 \mu$ s to $95 \mu$ s and has an enhanced contact effect with the dual mass-spring system in the MEMS inertial switch. The fabricated switches were tested by a shock test device. The results show that the switch can be reliably closed in all directions under the applied shock acceleration and has a long contact time, which is basically in accordance with the theoretical results.
\end{abstract}

\section{Introduction}

Inertial switches based on microelectromechanical system (MEMS) usually sense shock acceleration by a movable proof mass, and the proof mass contacts a fixed electrode when the acceleration is over a given threshold. They are of great interest in many areas such as toys, accessories, automotive, military weapons, healthcare, and other shock monitoring applications [1-5].

Since Frobenius et al. presented a cantilever-type electromechanical switch in 1972 [6], many studies have been done with different mechanisms: latching switch, bistable switch, tunable threshold switch, and fluidic switch in order to improve contact effect and realize higher reliability [710]. Besides, Currano et al. reported a 3-axis acceleration switch [5] and Liu and Hao [11] and Lin et al. [12] proposed a MEMS passive universal inertial switch. Although these switches achieved multidirectional detection, they have a short contact time with a rigid contact method (traditional contact method of the inertial switch), which is not conducive to signal processing for the subsequent circuit. Cai et al. [3] developed a switch with a movable contact point, and the contact point was installed on top center of the proof mass, which undergoes the largest deformation [13]; as a result, the switch has prolonged the contact time. Matsunaga and Esashi reported an acceleration switch with a dual massspring system and squeeze film effect is utilized to extend the contact time [2]. In addition, an electrostatic pull-in phenomenon was used to enable the switch to have an "on"state latching ability reported by Jia et al. [14] and Yang et al. [15] designed a microswitch with an electrophoretic flexible composite fixed electrode, which can realize a flexible contact between the electrodes and prolong the contact time; also, Lee et al. proposed a switch with extended contact time using carbon nanotube- (CNT-) contact pads [16]. Although the above-mentioned switches have a long contact time and an enhanced contact effect by changing the structure or using composite material, the influence factor of contact time by the theoretical analysis to guide the design of the switch was absent.

The closure characteristics of the switch include the contact time, contact effect, and switch-on capability in all directions. And the contact time is an important performance parameter of a MEMS inertial switch when it is used for military weapons subjected to high shock acceleration and requiring small size in order to improve reliability and 


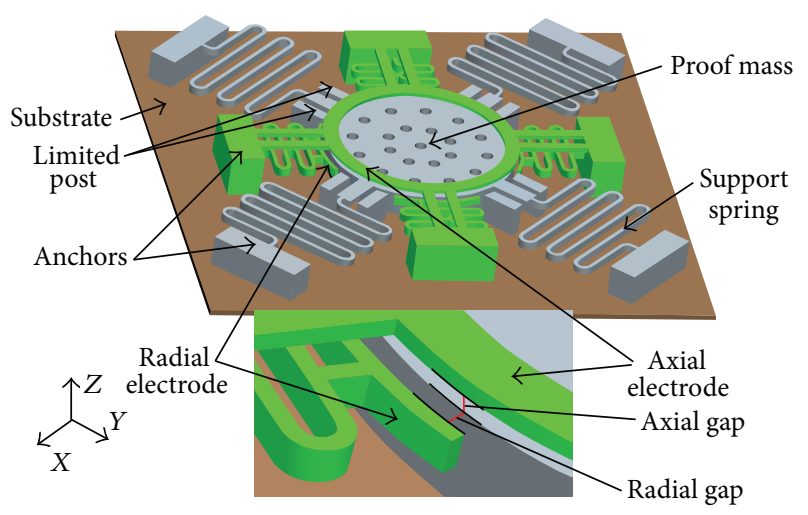

FIGURE 1: Schematic view of the proposed inertial switch.

miniaturize. As the contact time is short, it would not be conducive to signal processing for the subsequent circuit. In our previous paper, we proposed a novel MEMS omnidirectional inertial switch with flexible electrodes, which has omnidirectional sensitivities and enhanced contact effect. However, the theoretical method for calculating the contact time of the switch was not given [17]. In this paper, a preliminary method for calculating contact time of the dual mass-spring system in the inertial switch was presented and the simulation results verified the theoretical calculations; meanwhile, the theoretical results coincide with the test results. This could provide a theoretical basis for prolonged contact time of MEMS inertial switch.

\section{Device Design and Theoretical Analysis}

2.1. Device Configuration. The structure of the MEMS omnidirectional inertial switch is illustrated in Figure 1. It consists of two main parts: a thick suspended proof mass with springs as a movable electrode, arc-shaped strips with springs symmetrically around the proof mass, and a circle loop with a crossbeam above the proof mass as radial and axial stationary electrodes that are suspended by springs and cross beam. The movable electrode has a gap from the stationary electrodes that keep the circuit off in a static state. As for shock acceleration acting along any radial directions (in the $X O Y$ plane) or axial direction ( $Z$-axis direction), the movable electrode will move towards one of the stationary electrodes. When the acceleration exceeds the threshold value, the displacement of the movable electrode reaches the gap value, the movable electrode contacts the stationary electrodes, and the switch is turned on. As a result, a flexible contact between the movable electrode and stationary electrodes is realized by using flexible electrodes and the switch has a long contact time far greater than that of the literature $[11,12]$ with rigid contact. Also the use of flexible electrodes makes the switchon state more stable.

2.2. Theoretical Analysis of the Contact Time. The switch is mainly composed of mass-spring system and electrodespring system, which form a dual mass-spring system. In Figure $2, \alpha$ and $\beta$ are selected for defining the direction

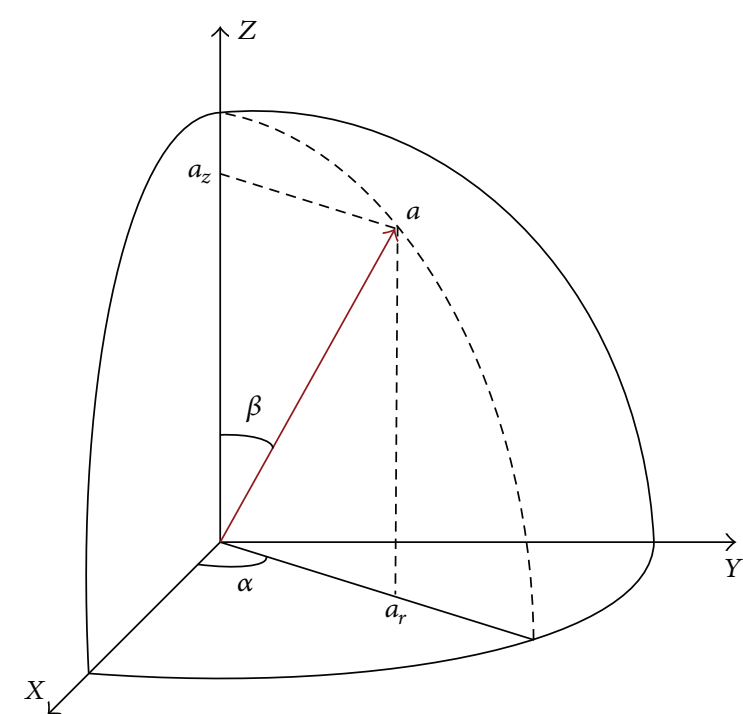

FIGURE 2: Schematic view of direction of shock acceleration.

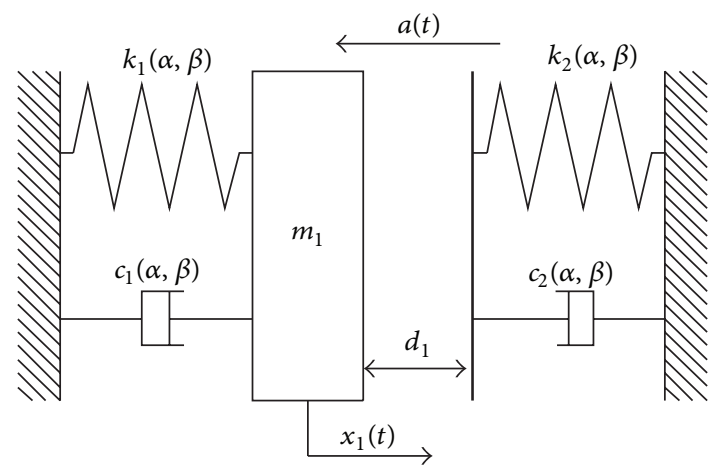

FIGURE 3: Physical model of the switch.

of shock acceleration in a half sphere. When the switch is subjected to shock acceleration $a(t)$ in $(\alpha, \beta)$ direction shown in Figure 2, the two systems simultaneously suffer the acceleration; then the contact between the mass and the electrode in this direction can be simplified to a massspring system colliding with an elastic wall. The mass of the electrode-spring system is so small that can be ignored comparing with the mass of the mass-spring system. Hence, the electrode-spring system is equivalent to an elastic wall with elastic coefficient and without mass. The dual mass-spring system can be equilibrium to the physical model of Figure 3, and the gap between electrodes is $d_{1}$ in initial state. $m_{1}$ is equivalent mass of the total mass of the proof mass and the support springs. $k_{1}(\alpha, \beta), k_{2}(\alpha, \beta)$ are system stiffness (seen in Section 3.1) of the mass-spring system and electrode-spring system in $(\alpha, \beta)$ direction, respectively. $c_{1}(\alpha, \beta), c_{2}(\alpha, \beta)$ are damping coefficient of the mass-spring system and electrodespring system in $(\alpha, \beta)$ direction, respectively.

The nonlinear of the stiffness is neglected because of small deformation of the two systems; as a result, $k_{1}(\alpha, \beta),\left(k_{2}(\alpha, \beta)\right)$ are a constant in a certain direction, while changing with $\alpha$ and $\beta$. 
(1) When $x_{1}(t)<d_{1}$, the proof mass does not collide with the elastic wall, so the proof mass dynamics is expressed as

$$
m_{1} \ddot{x}_{1}(t)+c_{1} \dot{x_{1}}(t)+k_{1} x_{1}(t)=m_{1} a(t),
$$

where $x_{1}(t)$ represents the displacement of the proof mass and $a(t)$ is the external shock acceleration and $a(t)=$ $a_{0} \sin \omega_{0} t\left(0 \leq t \leq \pi / \omega_{0}\right)$. Since the squeeze film damping and synovial damping of the designed switch are extremely small, the damping coefficient of $c_{1}$ and $c_{2}$ is ignored in order to simplify the calculation. Equation (1) is rewritten as follows:

$$
m_{1} \ddot{x}_{1}(t)+k_{1} x_{1}(t)=m_{1} a_{0} \sin \omega_{0} t
$$

By solving (2), we have the displacement $x_{1}(t)$ of the proof mass:

$$
x_{1}(t)=\frac{a_{0}}{\omega_{1}^{2}-\omega_{0}^{2}}\left(\sin \omega_{0} t-\frac{\omega_{0}}{\omega_{1}} \sin \omega_{1} t\right),
$$

where $\omega_{1}$ is the undamped resonant frequency of the proof mass and $\omega_{1}=\sqrt{k_{1} / m_{1}}$.

(2) When $x_{1}(t) \geq d_{1}$, the proof mass collides with the elastic wall and there is a pair of interaction forces between them, and $t$ of $x_{1}(t)=d_{1}$ (the interaction forces first appear) is the initial time of collision; the coupling equation of the proof mass and the elastic wall moving together is expressed as follows:

$$
\begin{gathered}
m_{1} \ddot{x}_{c}(t)+k_{1} x_{c}(t)+k_{2}\left[x_{c}(t)-d_{1}\right] \\
=m_{1} a_{0} \sin \omega_{0}\left(t+t_{r}\right),
\end{gathered}
$$

where $x_{c}(t)$ is displacement of the two electrodes moving together and $t$ is the time of the two electrodes moving together and $t_{r}$ is determined by $x_{1}\left(t_{r}\right)=d_{1}$. By solving (4) with the initial conditions of $x_{c}(0)=d_{1}, \dot{x}_{c}(0)=v_{0}=\dot{x}_{1}\left(t_{r}\right)$, one obtains

$$
\begin{aligned}
x_{c}(t)= & \frac{a_{0}}{\omega^{2}-\omega_{0}^{2}} \sin \omega_{0}\left(t+t_{r}\right) \\
& +\left(\frac{v_{0}}{\omega}-\frac{\omega_{0}}{\omega} \frac{a_{0}}{\omega^{2}-\omega_{0}^{2}} \cos \omega_{0} t_{r}\right) \sin \omega t \\
& +\left(\frac{\omega_{1}^{2}}{\omega^{2}} d_{1}-\frac{a_{0}}{\omega^{2}-\omega_{0}^{2}} \sin \omega_{0} t_{r}\right) \cos \omega t+\frac{\omega^{2}-\omega_{1}^{2}}{\omega^{2}} d_{1},
\end{aligned}
$$

where $\omega$ is given by $\omega=\sqrt{\left(k_{1}+k_{2}\right) / m_{1}}$. The displacement $x_{0}(t)$ of the proof mass and elastic wall moving together for the first collision is then expressed as

$$
x_{0}(t)=x_{c}(t)-d_{1}
$$

(3) When the displacement of the proof mass rebounding is equal to $d_{1}$, the two electrodes will begin to separate. The contact time of the first collision is the time of the proof mass and the elastic wall from collision to the separation process, which is the smallest positive root determined by the following formula:

$$
x_{c}\left(t_{c}\right)=d_{1}
$$

Then by solving (5) and (7), one obtains,

$$
\begin{aligned}
& \frac{m_{1} a_{0}}{k_{1}+k_{2}-m_{1} \omega_{0}^{2}} \sin \omega_{0}\left(t_{c}+t_{r}\right)+\sqrt{\frac{m_{1}}{k_{1}+k_{2}}} \\
& \cdot\left(v_{0}-\frac{m_{1} a_{0} \omega_{0}}{k_{1}+k_{2}-m_{1} \omega_{0}^{2}} \cos \omega_{0} t_{r}\right) \sin \sqrt{\frac{k_{1}+k_{2}}{m_{1}}} t_{c} \\
& +\left(\frac{k_{1} d_{1}}{k_{1}+k_{2}}-\frac{m_{1} a_{0}}{k_{1}+k_{2}-m_{1} \omega_{0}^{2}} \sin \omega_{0} t_{r}\right) \cos \sqrt{\frac{k_{1}+k_{2}}{m_{1}}} t_{c} \\
& -\frac{k_{1} d_{1}}{k_{1}+k_{2}}=0 .
\end{aligned}
$$

Hence, the contact time $t_{c}$ can be derived from (8). Also, (3), (6), and (8) are applicable to radial directions and axial direction by changing the value of $k_{1}(\alpha, \beta), k_{2}(\alpha, \beta)$, and $d_{1}$.

The solutions to (8) were obtained by using the MATLAB numerical method. The structural parameters were shown in our previous paper [17]; the difference is that $d_{1}$ in this paper is $20 \mu \mathrm{m}$, not $25 \mu \mathrm{m}$. According to (8), the contact time $t_{c}$ in $(\alpha, \beta)$ direction is related to the system stiffness $k_{1}(\alpha, \beta)$ of the mass-spring system in $(\alpha, \beta)$ direction, the system stiffness $k_{2}(\alpha, \beta)$ of the electrode-spring system in $(\alpha, \beta)$ direction, the mass $m_{1}$ of the proof mass, and the radial gap $d_{1} . \alpha=45^{\circ}$ and $\beta=90^{\circ}$ are selected for analyzing the influence of relevant parameters on the contact time, and the corresponding curves are shown in Figure 4.

As the stiffness $k_{2}$ decreases, the contact time $t_{c}$ increases rapidly, as shown in Figure 4(b). And it is shown in Figure $4(\mathrm{~d})$ that the contact time $t_{c}$ increases significantly with the mass $m_{1}$. In addition, the contact time $t_{c}$ increases slowly as the system stiffness $k_{1}$ and gap $d_{1}$ decrease within the range of enabling the switch to close, as shown in Figures 4(a) and 4(c).

\section{Simulations and Analysis}

3.1. The System Stiffness in All Directions. The system stiffness is the stiffness of the mass-spring system in the switch, including the proof mass and four springs. Although the stiffness of a single spring can be calculated by analytical analysis easily, the system stiffness $\left(k_{1}(\alpha, \beta)\right)$ of the present device in all directions cannot be calculated conveniently. So the FE method is used to analyze the system stiffness. Figures 5(a) and 5(b) show a stiffness distribution of the switch in the $X O Y$ plane and $X O Z$ plane, respectively. As a result, the system stiffness is approximately uniform in the $X O Y$ plane, where the error rate between the maximum stiffness and the minimum stiffness is only $7 \times 10^{-8}$. Obviously, it is indicated that the system stiffness in $Z$-axis direction is a minimum in the system stiffness according to Figure 5(b), 


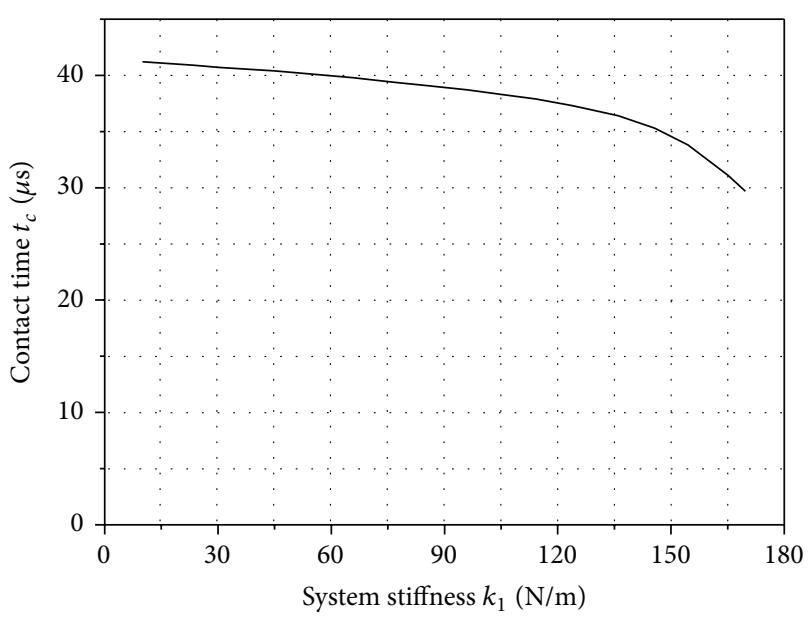

(a)

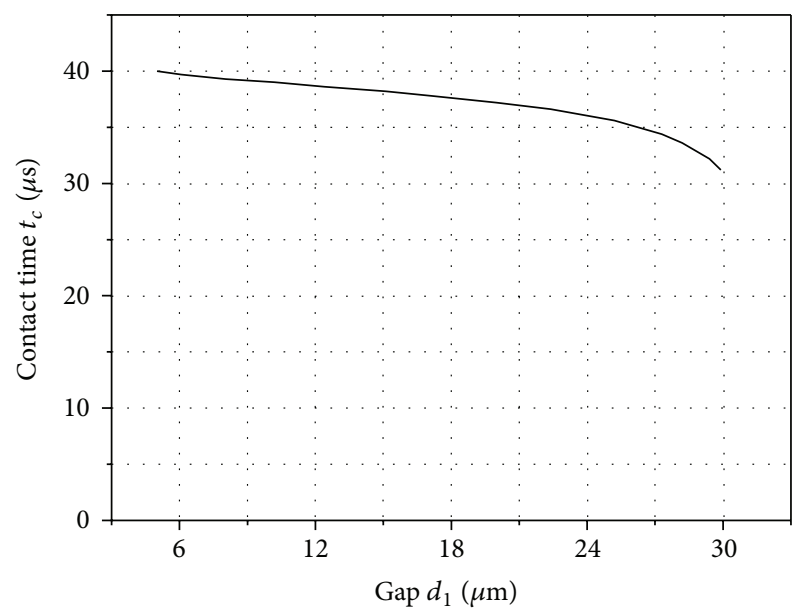

(c)

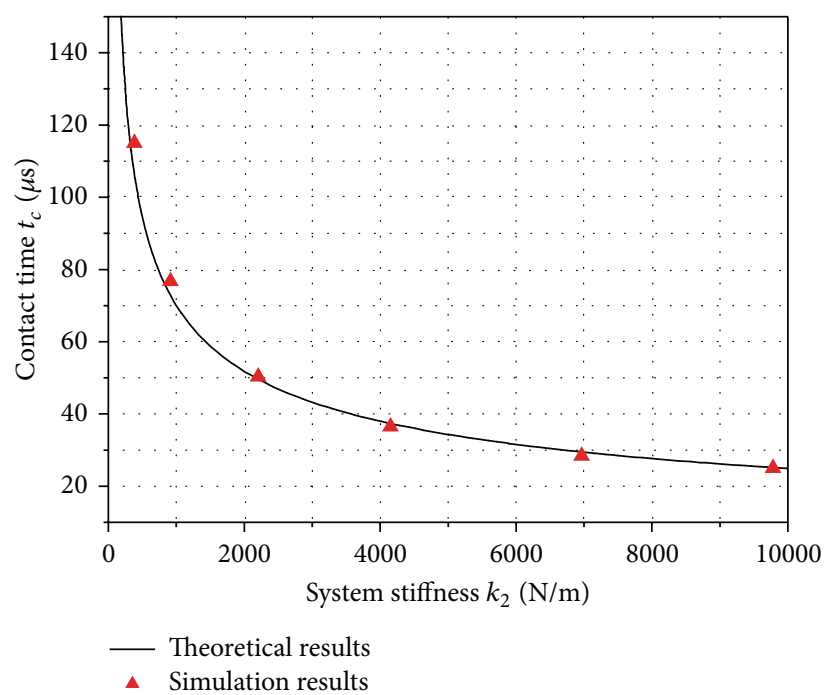

(b)

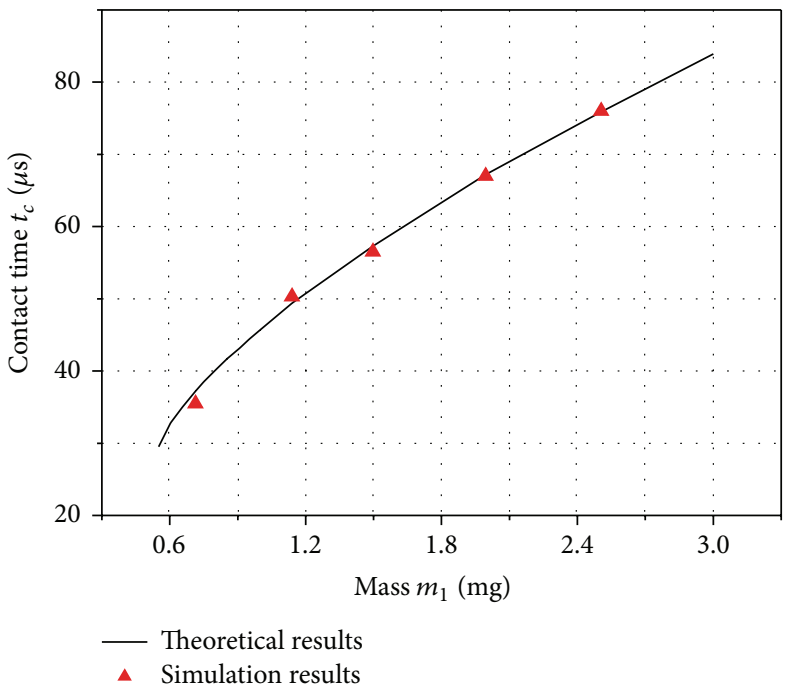

(d)

Figure 4: The curves of (a) the contact time versus system stiffness $k_{1}$, (b) the contact time versus system stiffness $k_{2}$, (c) the contact time versus gap $d_{1}$, and (d) the contact time versus mass $m_{1}$.

and the error rate between the maximum stiffness and the minimum stiffness in all directions is $8.8 \%$. Although this error can be reduced by adjusting the parameters of the structure, it cannot be eliminated for the single mass-spring system. Furthermore, it is desirable that the system stiffness in all directions is uniform for the omnidirectional switch, so we can adjust the radial gap and axial gap appropriately in order to obtain better omnidirectional sensitivity of the switch. Besides, the system stiffness increases gradually as the angle with the $Z$-axis increases in the $X O Z$ plane.

3.2. Influence of Structural Parameters on the Stiffness. The mass-spring system is subjected to greater shock and vibration in practical applications due to the switch driven by inertial force; as a result, analysis of the system stiffness of the mass-spring system under dynamic load has more engineering significance. However, displacements of the electrode-spring systems with extremely small mass and large stiffness are almost zero under dynamic load. These displacements are induced by force of collision between the proof mass and electrodes, so analysis of the stiffness of electrode-spring systems under static load is more accurate. The stiffness of the switch was simulated using the method of changing a single factor. The simulation results are shown in Figure 6.

Figure 6(a) indicates that the system stiffness $k_{1}$ of massspring system increases with width of spring and the stiffness $k_{2}$ of radial electrode increases with width of spring similarly. It is indicated that the stiffness is most sensitive to the spring width; as the width has a small variation, the stiffness will have greater change. Besides, the stiffness $k_{2}$ of axial electrode increases rapidly with thickness of the cross beam, while 


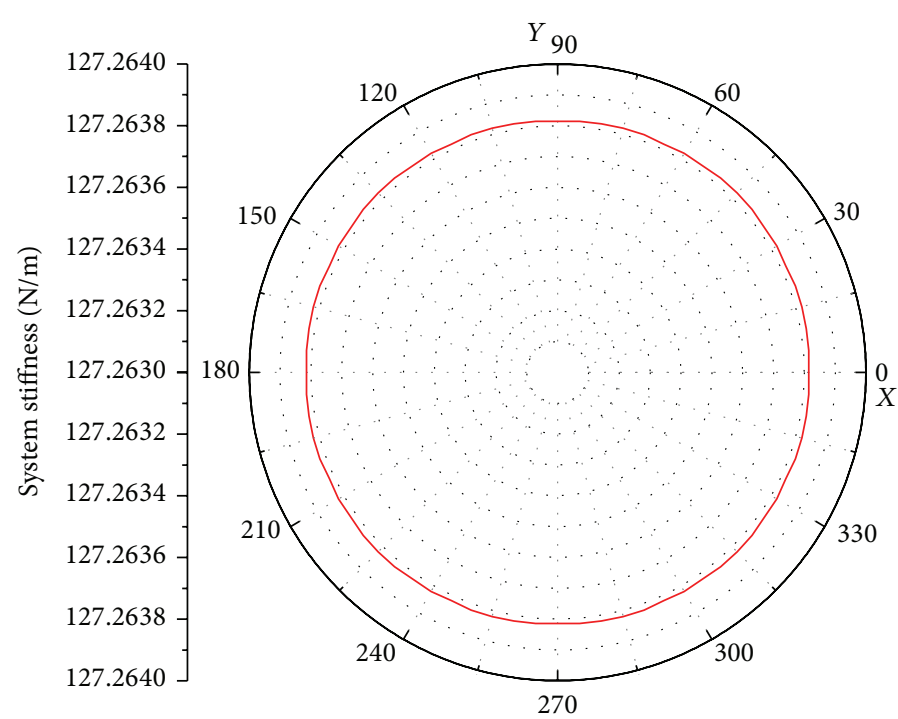

(a)
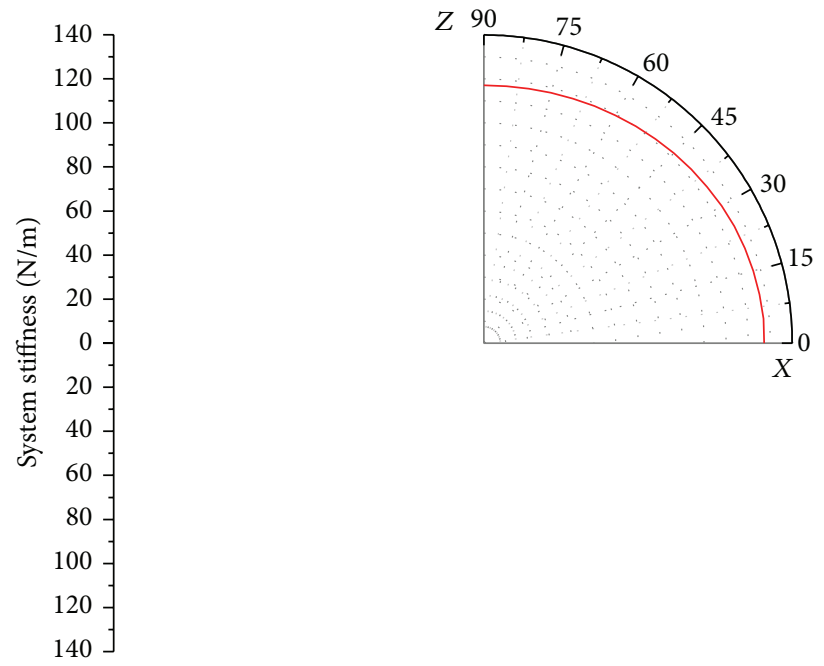

FIGURE 5: Simulated system stiffness distributions of the switch in the (a) XOY plane and (b) XOZ plane.

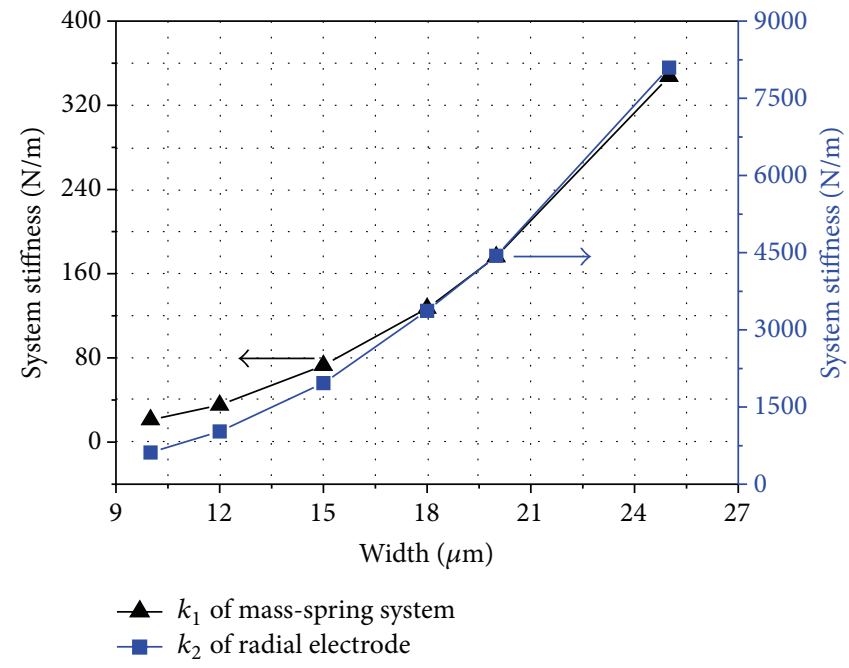

(a)

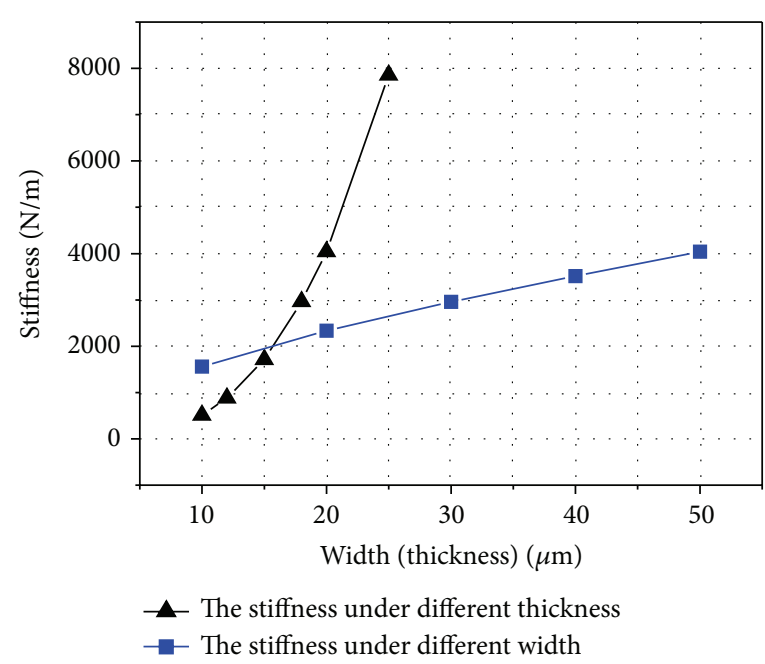

(b)

Figure 6: The curve of the stiffness versus the structural parameters: (a) the stiffness of mass-spring system and radial electrode under different spring width and (b) the stiffness of axial electrode under different crossbeam width and thickness.

increasing slowly with width of the cross beam, as shown in Figure 6(b).

3.3. Dynamic Contact Simulation. ANSYS software was applied to construct the finite element model and a simulation model with two radial electrodes and axial electrode is employed, as shown in Figure 7. The material is nickel, where Young's modulus is $180 \mathrm{GPa}$ and Poisson's ratio is 0.3 [18]. SWEEP method was used to mesh the model and the grid of contact area is made to have high quality. Finally, the displacements of outer end of the springs and the crossbeam are constrained to be zero at all degrees of freedom. And herein radial gap and axial gap are $20 \mu \mathrm{m}$ and $30 \mu \mathrm{m}$, respectively.

In order to clearly illustrate the dynamic impact process, according to our application environment an over-threshold half-sine acceleration with amplitude $450 \mathrm{~g}$ and duration $1 \mathrm{~ms}$ was applied to the model in different shock directions, such as $X$-axis direction, $45^{\circ}$ with the $X$-axis direction in the $X O Y$ plane, $Z$-axis direction, and $45^{\circ}$ with the $Z$-axis direction in the $X O Z$ plane. The simulation results are shown in Figure 8, including the displacements of the proof mass 


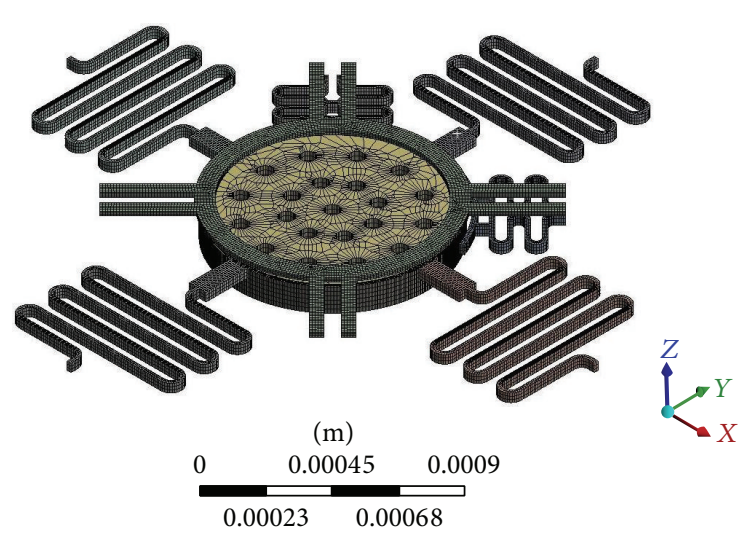

FIGURE 7: FE model of the switch.

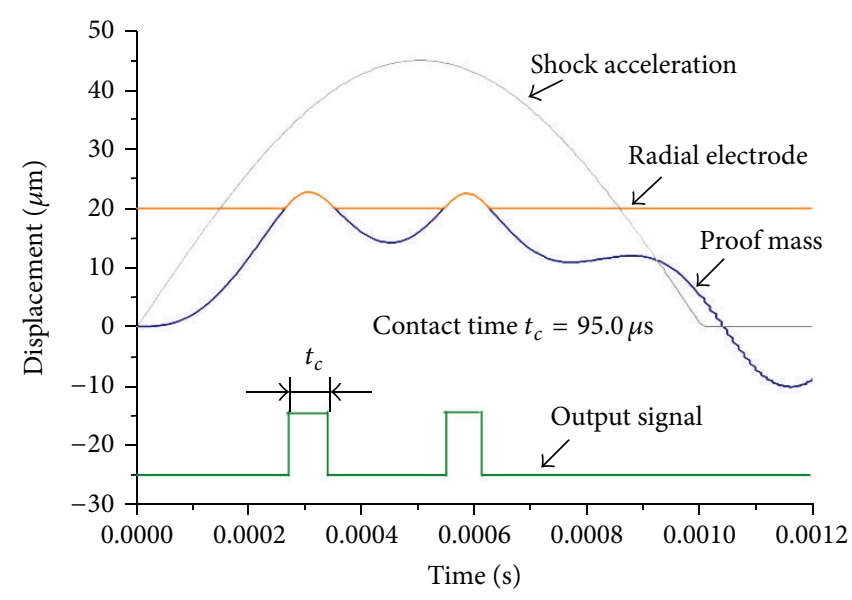

(a)

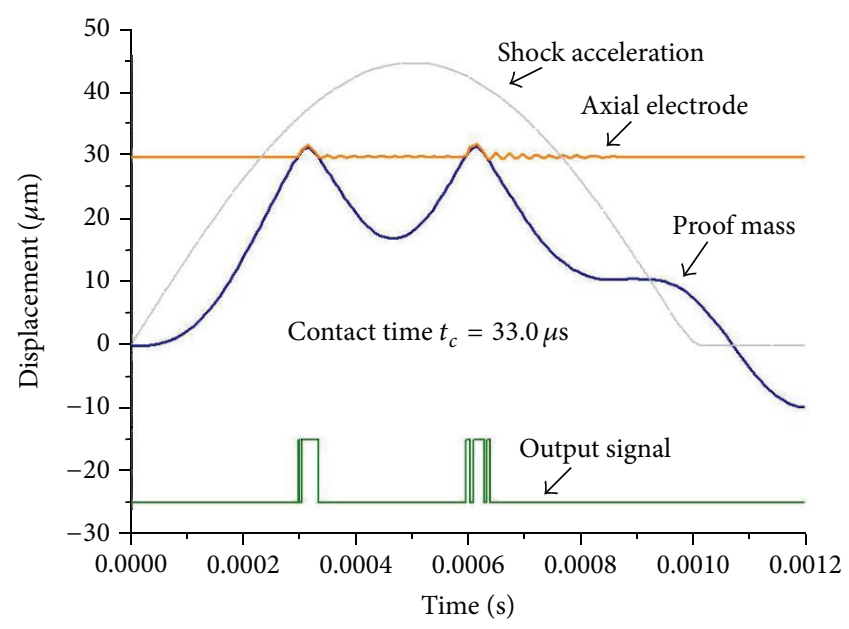

(c)

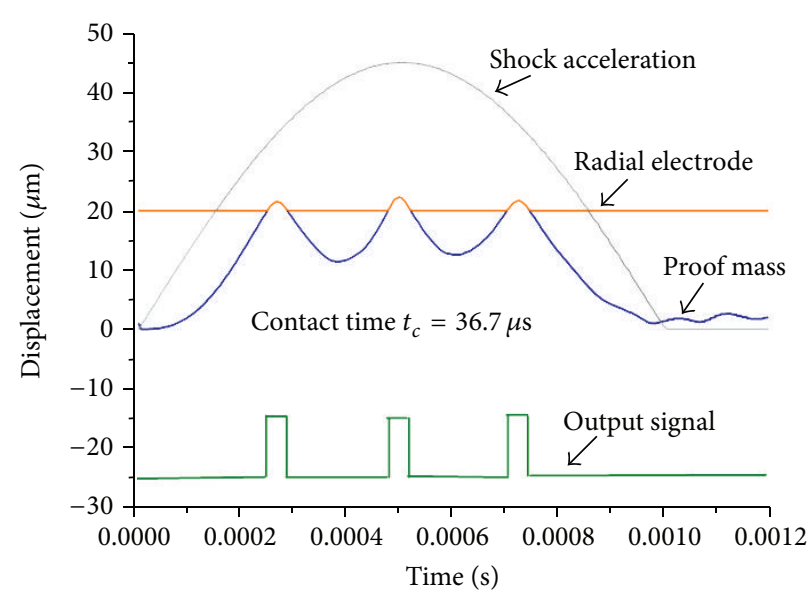

(b)

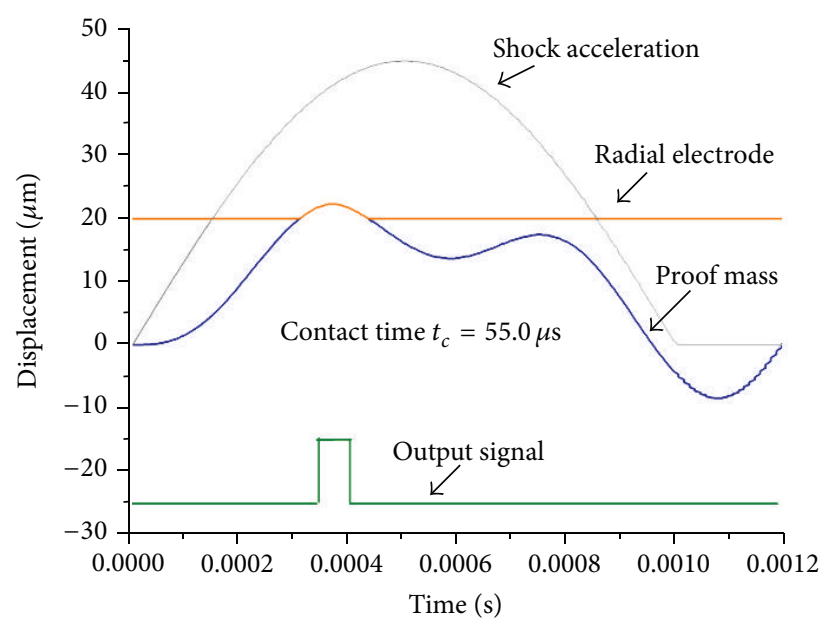

(d)

FIGURE 8: Dynamic simulation results of the switch in the direction of (a) $X$-axis direction, (b) $45^{\circ}$ with the $X$-axis direction in the $X O Y$ plane, (c) $Z$-axis direction, and (d) $45^{\circ}$ with the $Z$-axis direction in the $X O Z$ plane.

and the electrodes, as well as shock acceleration signal and output signal.

In Figure 8(a), when the displacement of the proof mass reaches the radial gap $20 \mu \mathrm{m}$, the proof mass contacts with the radial electrodes, and the external circuit is switched on, so we obtain the output signal. The contact time of the switch is $95.0 \mu$ s in $X$-axis direction. Similarly, the contact time of the switch in $45^{\circ}$ with the $X$-axis direction in the XOY plane is $40 \mu \mathrm{s}$, as seen in Figure 8(b). It is shorter than that in $X$-axis direction, which results from the system stiffness 


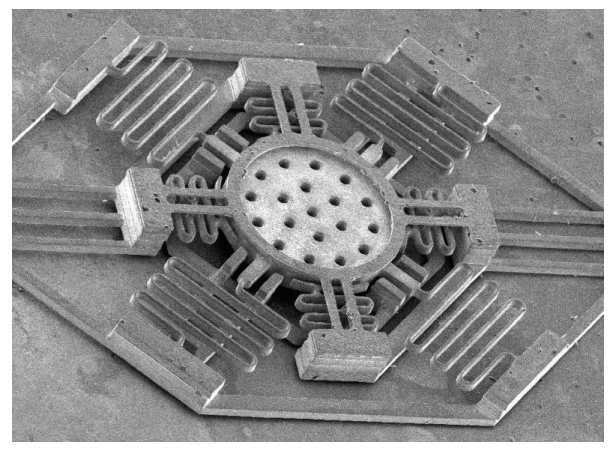

FIGURE 9: SEM image of the switch prototypes.

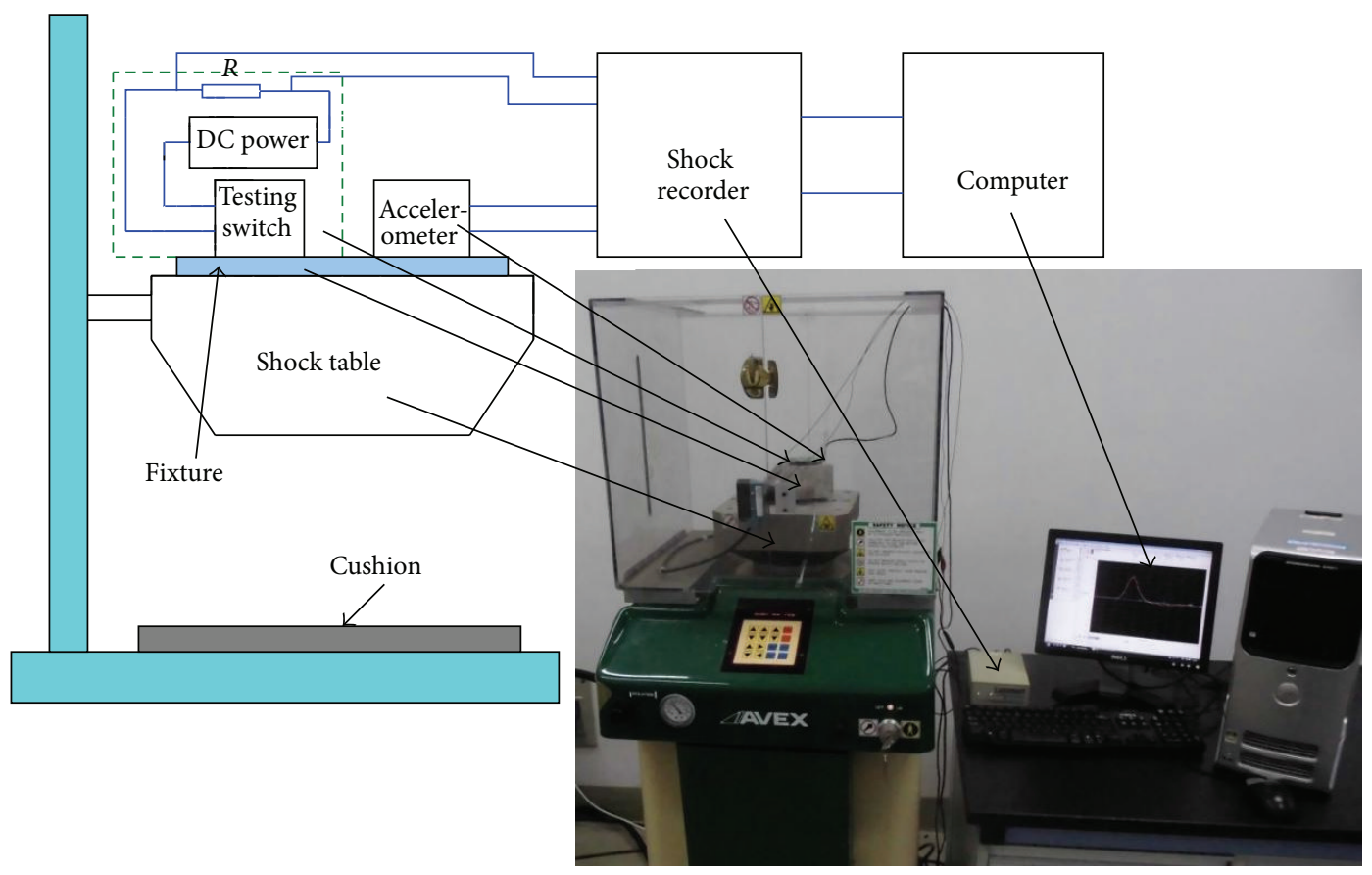

FIGURE 10: Shock test devices for the switch prototypes.

of radial electrode in $45^{\circ}$ with the $X$-axis direction greater than that in $X$-axis direction. This situation is consistent with the theoretical results shown in Figure 4(b). Also, it can be seen from Figures 8(c) and 8(d) that the contact time in $Z$ axis direction and $45^{\circ}$ with the $Z$-axis direction in the $X O Z$ plane is $33 \mu \mathrm{s}$ and $55 \mu \mathrm{s}$, respectively. In the $45^{\circ}$ with the $Z$-axis direction, the proof mass simultaneously generates displacement in $X$-axis direction and $Z$-axis direction. The displacement in $X$-axis direction is greater than the radial gap, while the displacement in $Z$-axis direction is smaller than the axial gap; as a result, the proof mass contacts with the radial electrode, not the axial electrode. It is indicated that the switch can be reliably closed under the applied shock acceleration of $450 \mathrm{~g}$ and has a long contact time.

The contact time $t_{c}$ can be obtained by changing the electrode spring width $(W)$ in the simulation model, while other parameters of the switch remain the same. A series of points $\left(k_{2}, t_{c}\right)$ were derived from $\left(W, t_{c}\right)$ and are marked in
Figure 4(b) as simulation results. Similarly, a series of points $\left(m_{1}, t_{c}\right)$ were obtained by changing the proof mass thickness and are marked in Figure 4(d). The results of simulation and theoretical calculation are consistent. The theoretical calculation can be used to predict the contact time of the designed switch.

\section{Testing}

The MEMS omnidirectional inertial switch was fabricated based on nonsilicon surface micromachining technology. The SEM image of the fabricated switch with a size of $2.8 \mathrm{~mm} \times$ $2.8 \mathrm{~mm} \times 210 \mu \mathrm{m}$ and minimum linewidth $15 \mu \mathrm{m}$ is shown in Figure 9 .

The fabricated switch prototypes were tested by a shock test system, where a standard accelerometer (CA-YD-180) with sensitivity of $5.292 \mathrm{mV} / \mathrm{g}$ was used for calibration and 


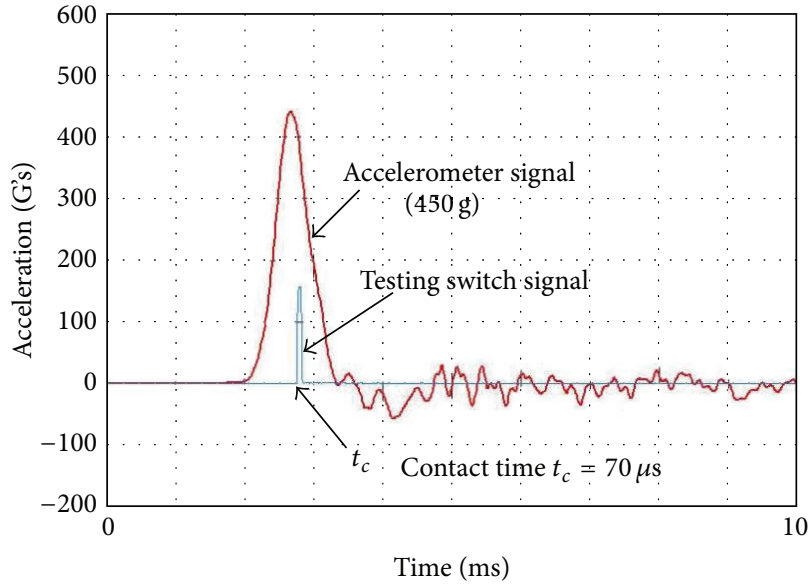

(a)

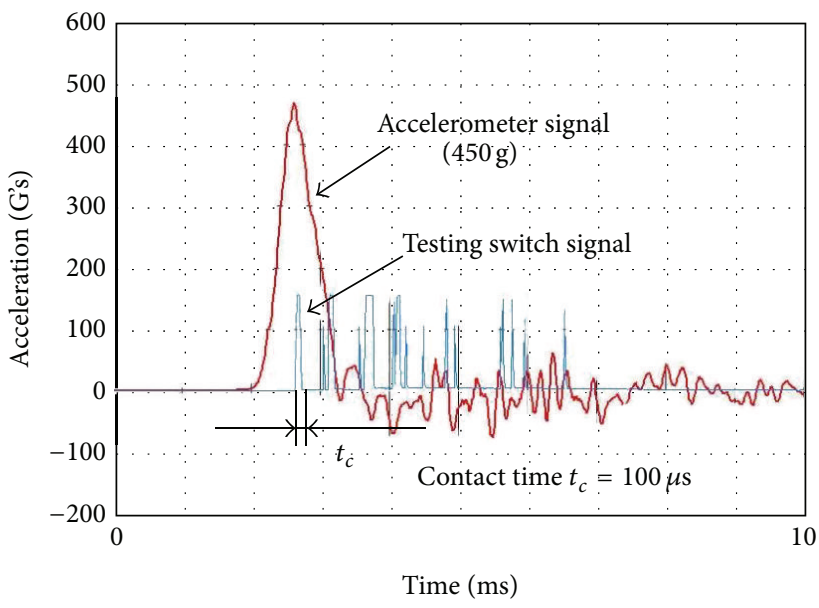

(c)

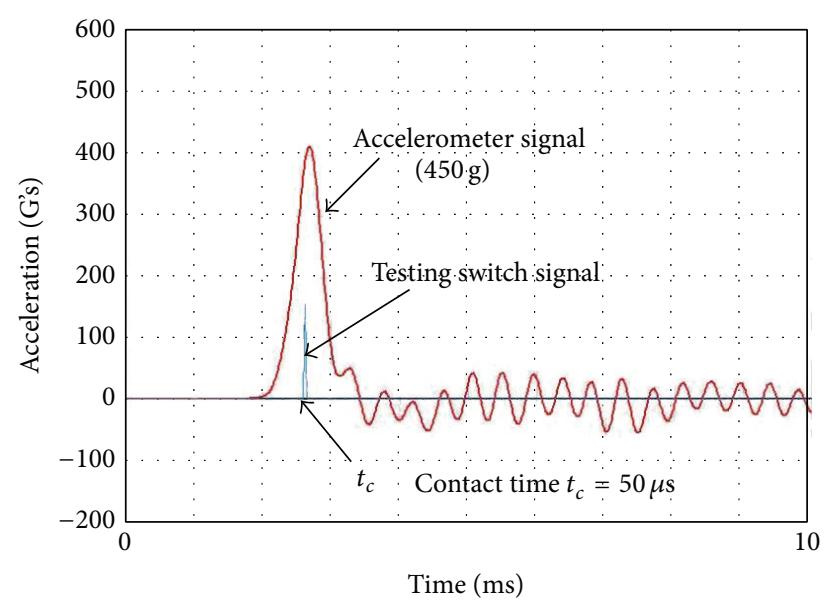

(b)

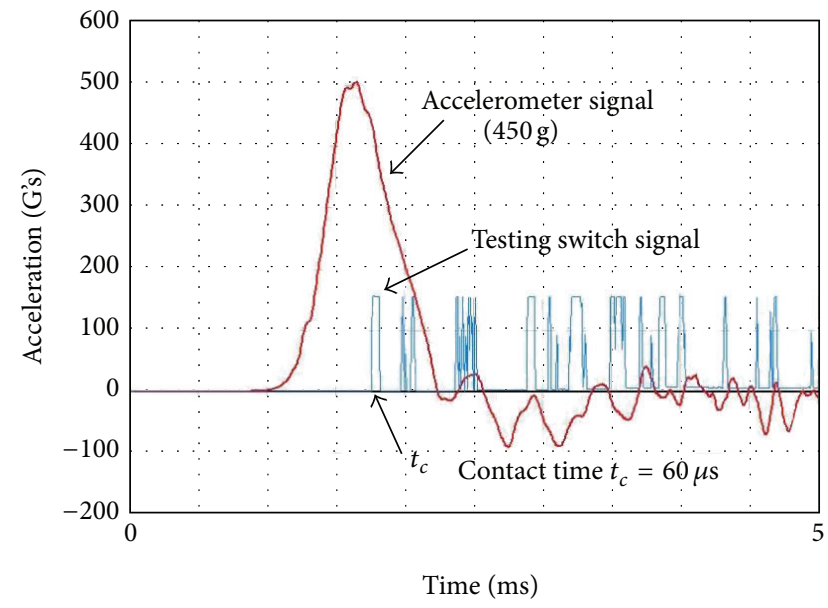

(d)

FIGURE 11: Shock test results of the switch in the direction of (a) $X$-axis direction, (b) $45^{\circ}$ with the $X$-axis direction in the $X O Y$ plane, (c) $Z$-axis direction, and (d) $45^{\circ}$ with the $Z$-axis direction in the $X O Z$ plane.

a voltage division circuit with DC supply voltage $3 \mathrm{~V}$ and load resistance $200 \Omega$ was employed. A data acquisition system was adopted to simultaneously capture the output of the accelerometer and monitor the voltage through the load resistance, as shown in Figure 10. Both the testing switch and the standard accelerometer were fixed on a special fixture, with their sensitive direction perpendicular to the ground. The different testing angle between switch and acceleration was achieved by the fixture. Half-sine accelerations with different amplitude and pulse width can be obtained by adjusting the shock table parameters.

As a comparison, a half-sine acceleration with amplitude about $450 \mathrm{~g}$ and duration $1 \mathrm{~ms}$ was applied to the switch in different shock directions, such as $X$-axis direction, $45^{\circ}$ with the $X$-axis direction in the $X O Y$ plane, $Z$-axis direction, and $45^{\circ}$ with the $Z$-axis direction in the $X O Z$ plane. The test results are shown in Figure 11.

It is shown in Figure 11 that the switch can be reliably turned on in radial directions, axial direction, and a certain angle with the axial direction under the applied half-sine acceleration with amplitude $450 \mathrm{~g}$ and duration $1 \mathrm{~ms}$, which is in agreement with the simulation results. Nevertheless, there is a little deviation on its contact time in test compared with the results of theoretical calculation, as shown in Table 1.

It can be seen from Table 1 that the results of simulation and theoretical calculation are basically consistent once again. However, the theoretical value and test value have an error rate of $24.7 \%, 34.0 \%$, and $175.5 \%$ in $X$-axis direction, $45^{\circ}$ with the $X$-axis direction in the XOY plane, and $Z$ axis direction, respectively. Based on the measured structural parameters of the switch prototype, the actual spring width is smaller than the designed one, the springs width of the proof mass and radial electrodes decrease by $4 \sim 5 \mu \mathrm{m}$ and $2 \sim 3 \mu \mathrm{m}$, respectively, and the thickness and width of the crossbeam decrease by $4 \sim 8 \mu \mathrm{m}$ and $6 \sim 10 \mu \mathrm{m}$, respectively, but the radial gap increases by $2 \sim 4 \mu \mathrm{m}$, as shown in Figure 12 (the distance between two red dashed lines represents the design width). When the spring width or crossbeam thickness is smaller, the stiffness decreases obviously as seen in Figure 6. As a result, the contact time increases, which is verified by Figure 4(b). 


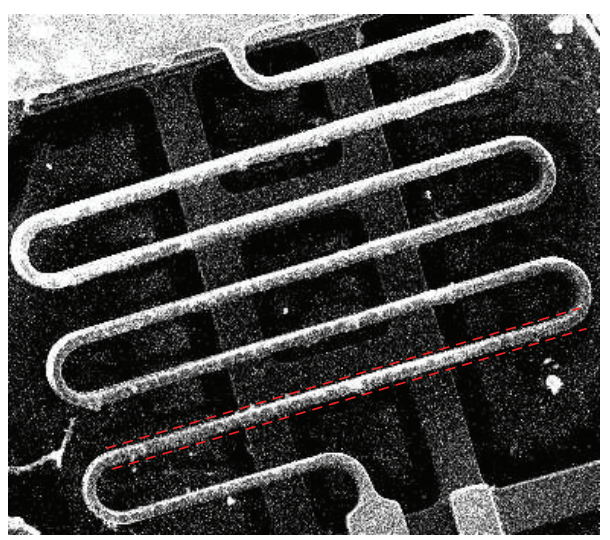

(a)

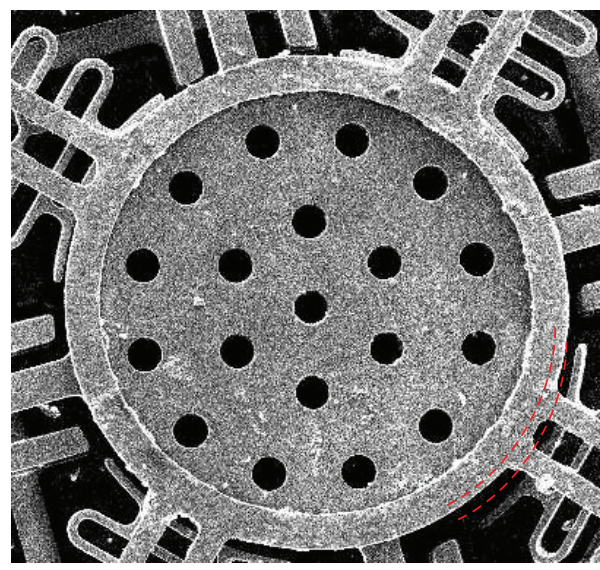

(c)

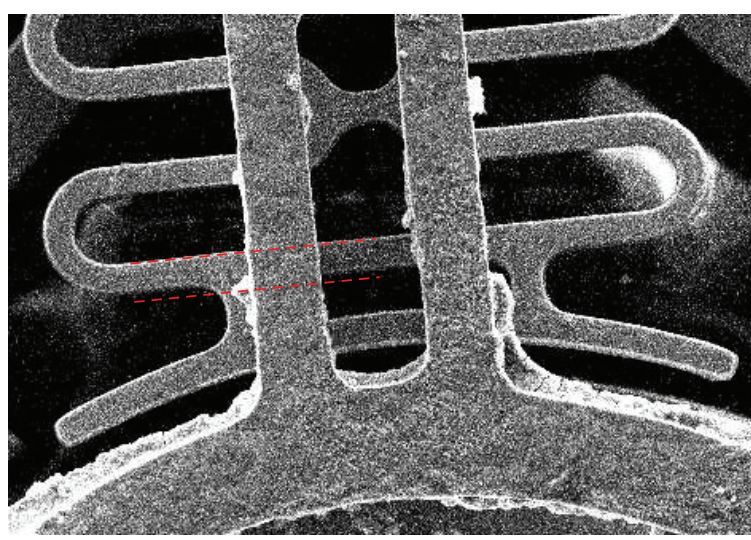

(b)

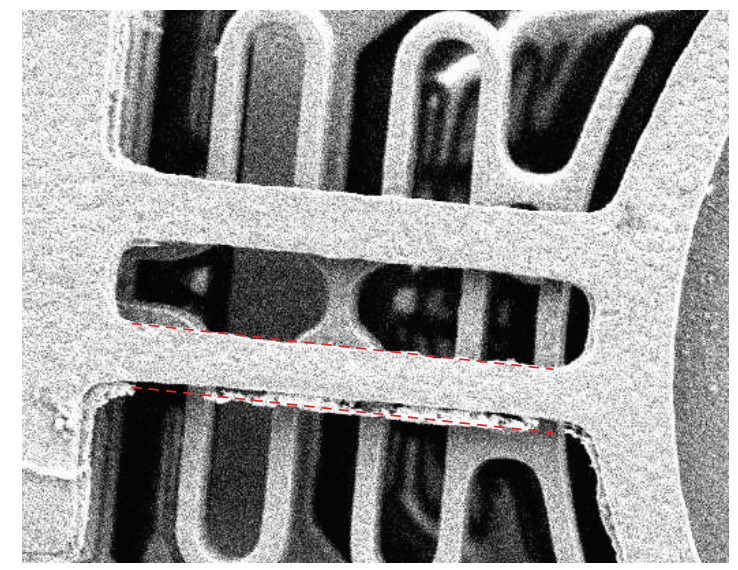

(d)

FIGURE 12: Fabrication errors of the switch (a) width of the proof mass spring decreasing, (b) width of the radial electrode spring decreasing, (c) radial gap increasing, and (d) width of the crossbeam decreasing.

TABLE 1: Theoretical value, simulation value, and test value of contact time in typical directions.

\begin{tabular}{|c|c|c|c|c|}
\hline Typical directions & $X$-axis direction & $\begin{array}{c}45^{\circ} \text { with the } X \text {-axis in the } \\
\text { XOY plane }\end{array}$ & $Z$-axis direction & $\begin{array}{c}45^{\circ} \text { with the } Z \text {-axis in the } \\
X O Z \text { plane }\end{array}$ \\
\hline Theoretical value $(\mu \mathrm{s})$ & 93.0 & 37.3 & 35.3 & 1 \\
\hline Simulated value $(\mu \mathrm{s})$ & 95.0 & 36.7 & 33.0 & 55.0 \\
\hline Test value $(\mu \mathrm{s})$ & 70 & 50 & 100 & 60 \\
\hline $\begin{array}{l}\text { Error rate of theoretical } \\
\text { value and simulation value }\end{array}$ & $2.1 \%$ & $1.6 \%$ & $6.9 \%$ & 1 \\
\hline $\begin{array}{l}\text { Error rate of theoretical } \\
\text { value and test value }\end{array}$ & $24.7 \%$ & $34.0 \%$ & $175.5 \%$ & 1 \\
\hline
\end{tabular}

This is considered to be the main reason of the contact time in test longer than theoretical value in $45^{\circ}$ with the $X$-axis direction in the $X O Y$ plane and $Z$-axis direction. In addition, in $X$-axis direction the spring width decreasing is not the only reason for the deviation, and as the radial gap increases, it brings about the contact time decreases shown in Figure 4(c); these cases may be the reason for the contact time decreasing in $X$-axis direction.

By modifying the model based on the measured parameters of the fabricated switch prototype, the test results coincide with the theoretical results. In Figures 11(c) and 11(d), a bounce phenomenon occurs, and it is necessary to further optimize the structural parameters for improving performance of the switch.

\section{Conclusion}

A preliminary method for calculating contact time of a dual mass-spring system applied to shock acceleration was proposed, the influence of relevant parameters on the contact time was analyzed, and the simulation results verified the theoretical calculations. The system stiffness of the mass-spring 
system in all directions was obtained by using the FE method and dynamic contact simulation results based on FEM in typical directions indicate that the switch has a long contact time and has an enhanced contact effect with the dual massspring system. The fabricated switches were tested by a shock test device. The test results show that the switch can be reliably closed in all directions under the applied shock acceleration of $450 \mathrm{~g}$ and has a contact time within the range of $50 \mu \mathrm{s}$ to $100 \mu \mathrm{s}$, which has a little deviation compared with the theoretical results. It is indicated that fabrication error is the main reason, especially error of spring width and crossbeam thickness and width.

\section{Conflict of Interests}

The authors declare that there is no conflict of interests regarding the publication of this paper.

\section{Acknowledgments}

This work is supported by National Natural Science Foundation of China (51475245).

\section{References}

[1] S. Michaelis, H.-J. Timme, M. Wycisk, and J. Binder, "Additive electroplating technology as a post-CMOS process for the production of MEMS acceleration-threshold switches for transportation applications," Journal of Micromechanics and Microengineering, vol. 10, no. 2, pp. 120-123, 2000.

[2] T. Matsunaga and M. Esashi, "Acceleration switch with extended holding time using squeeze film effect for side airbag systems," Sensors and Actuators A: Physical, vol. 100, no. 1, pp. 10-17, 2002.

[3] H. Cai, G. Ding, Z. Yang, Z. Su, J. Zhou, and H. Wang, "Design, simulation and fabrication of a novel contact-enhanced MEMS inertial switch with a movable contact point," Journal of Micromechanics and Microengineering, vol. 18, no. 11, Article ID 115033, 10 pages, 2008.

[4] G. L. Smith, “Triaxial MEMS acceleration switch,” US Patent 8,237,521, 2012.

[5] L. J. Currano, C. R. Becker, G. L. Smith, B. Isaacson, and C. J. Morris, "3-axis acceleration switch for traumatic brain injury early warning," in Proceedings of the 25th IEEE International Conference on Micro Electro Mechanical Systems (MEMS '12), pp. 484-487, Paris, France, January-February 2012.

[6] W. D. Frobenius, S. A. Zeitman, M. H. White, D. D. O'Sullivan, and R. G. Hamel, "Microminiature ganged threshold accelerometers compatible with integrated circuit technology," IEEE Transactions on Electron Devices, vol. 19, no. 1, pp. 37-40, 1972.

[7] Z. Y. Guo, Z. C. Yang, L. T. Lin et al., "Design, fabrication and characterization of a latching acceleration switch with multicontacts independent to the proof-mass," Sensors and Actuators A: Physical, vol. 166, no. 2, pp. 187-192, 2011.

[8] J. Zhao, Y. Yang, K. Fan, P. Hu, and H. Wang, "A bistable threshold accelerometer with fully compliant clamped-clamped mechanism," IEEE Sensors Journal, vol. 10, no. 5, pp. 1019-1024, 2010.
[9] H. Kim, Y. H. Jang, Y. K. Kim, and J. M. Kim, "MEMS acceleration switch with bi-directionally tunable threshold," Sensors and Actuators A: Physical, vol. 208, pp. 120-129, 2014.

[10] K. Yoo, U. Park, and J. Kim, "Development and characterization of a novel configurable MEMS inertial switch using a microscale liquid-metal droplet in a microstructured channel," Sensors and Actuators A: Physical, vol. 166, no. 2, pp. 234-240, 2011.

[11] S.-J. Liu and Y.-P. Hao, "Annular passive universal MEMS inertial switch," Journal of Chinese Inertial Technology, vol. 21, no. 2, pp. 240-244, 2013.

[12] H. F. Lin, H. T. He, Y. M. Bian, and Y. J. Yang, "Novel passive MEMS universal crash switch," MEMS Device and Technology, vol. 46, pp. 358-361, 2009.

[13] H. G. Cai, Z. Q. Yang, G. F. Ding, and H. Wang, "Development of a Novel MEMS Inertial Switch With a Compliant Stationary Electrode," IEEE Sensors Journal, vol. 9, no. 7, pp. 801-808, 2009.

[14] M. J. Jia, X. X. Li, Z. H. Song, M. H. Bao, Y. L. Wang, and H. Yang, "Micro-cantilever shocking-acceleration switches with threshold adjusting and 'on'-state latching functions," Journal of Micromechanics and Microengineering, vol. 17, no. 3, pp. 567$575,2007$.

[15] Z. Yang, B. Zhu, W. Chen, G. Ding, H. Wang, and X. Zhao, "Fabrication and characterization of a multidirectional-sensitive contact-enhanced inertial microswitch with a electrophoretic flexible composite fixed electrode," Journal of Micromechanics and Microengineering, vol. 22, no. 4, Article ID 045006, 2012.

[16] J.-I. Lee, Y. Song, H. Jung, J. Choi, Y. Eun, and J. Kim, "Deformable carbon nanotube-contact pads for inertial microswitch to extend contact time," IEEE Transactions on Industrial Electronics, vol. 59, no. 12, pp. 4914-4920, 2012.

[17] Z. Xi, P. Zhang, W. Nie, L. Du, and Y. Cao, "A novel MEMS omnidirectional inertial switch with flexible electrodes," Sensors and Actuators A: Physical, vol. 212, pp. 93-101, 2014.

[18] K. J. Hemker and H. Last, "Microsample tensile testing of LIGA nickel for MEMS applications," Materials Science and Engineering A, vol. 319-321, pp. 882-886, 2001. 

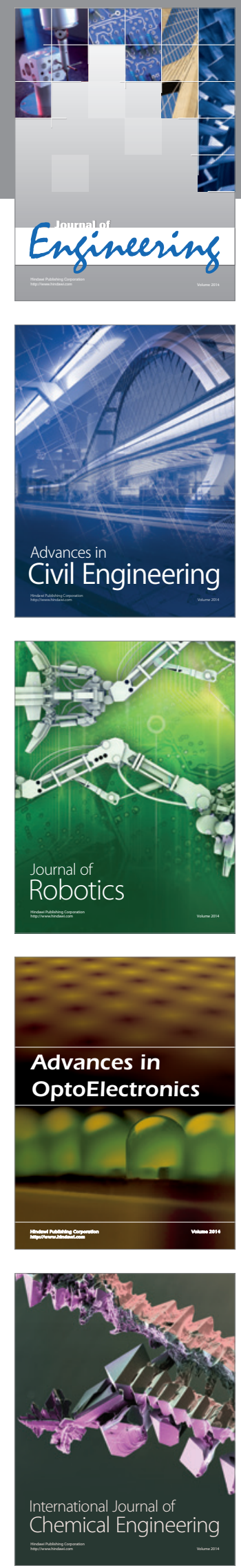

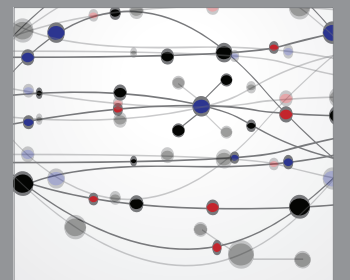

The Scientific World Journal
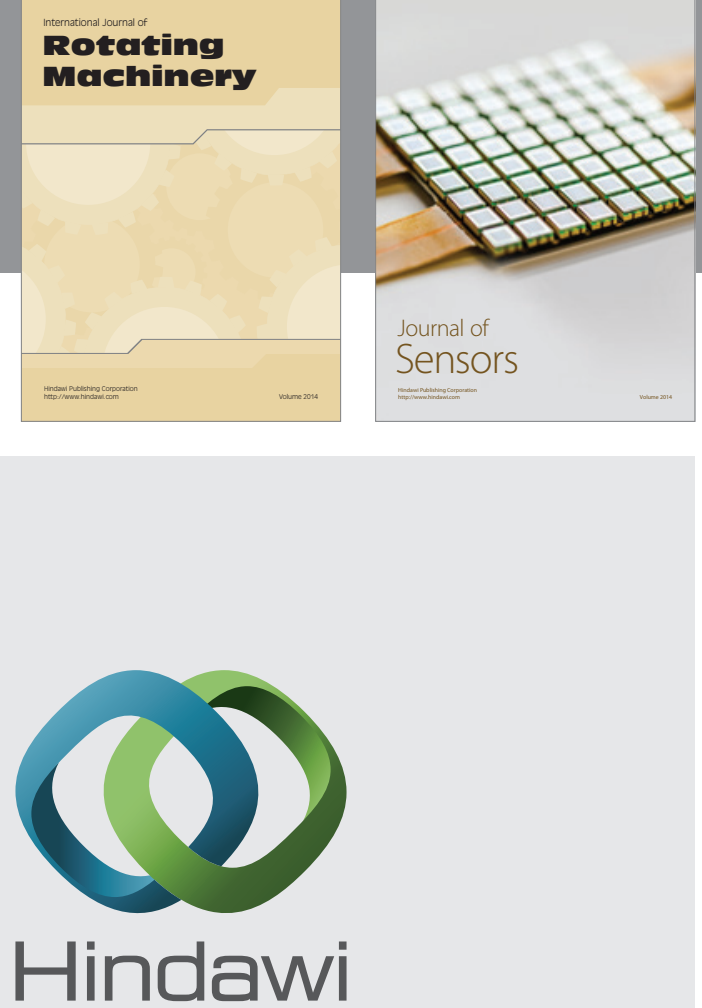

Submit your manuscripts at http://www.hindawi.com
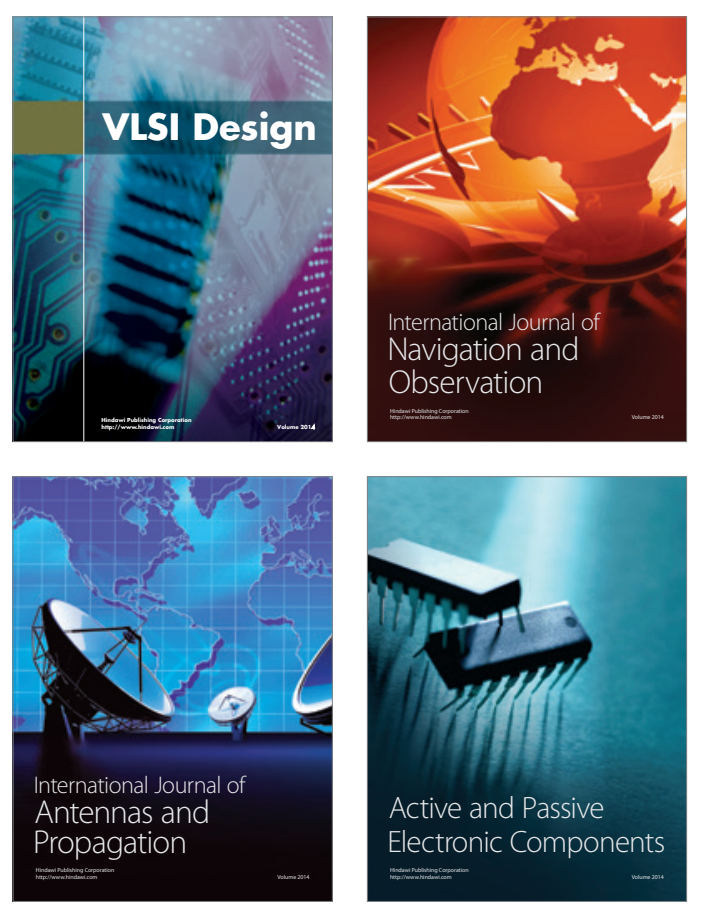
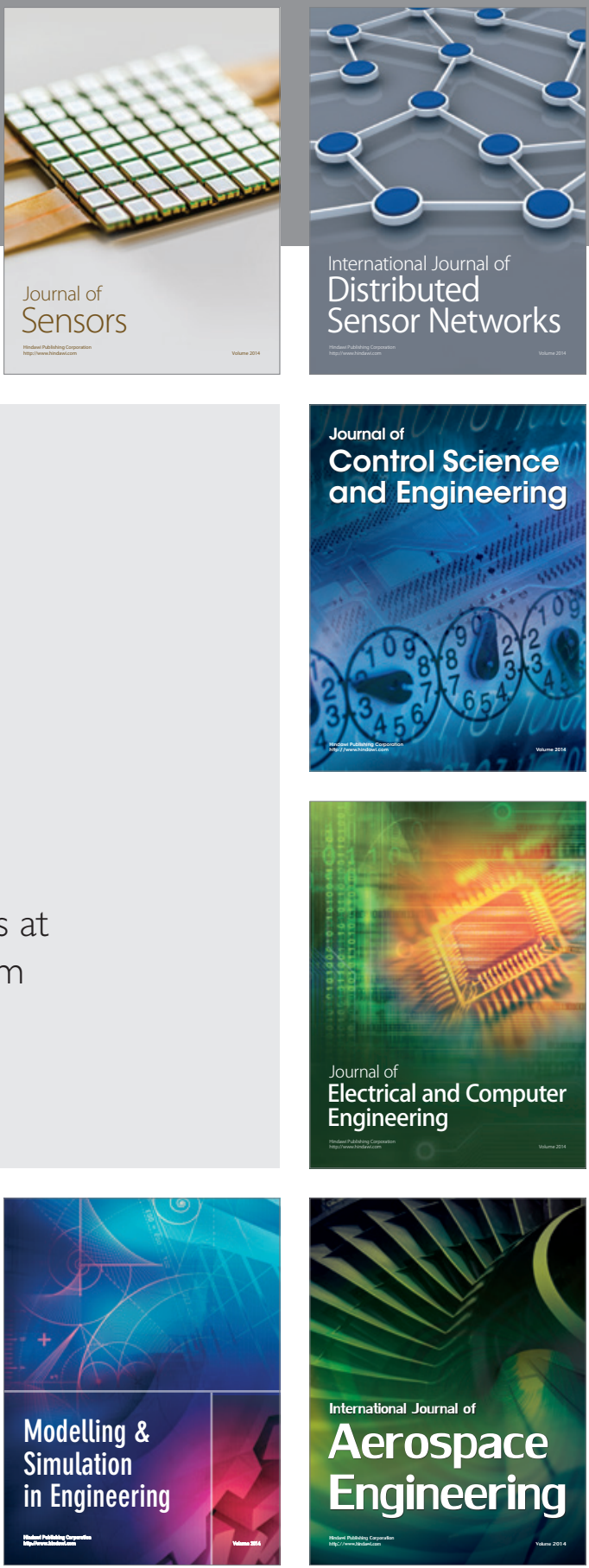

Journal of

Control Science

and Engineering
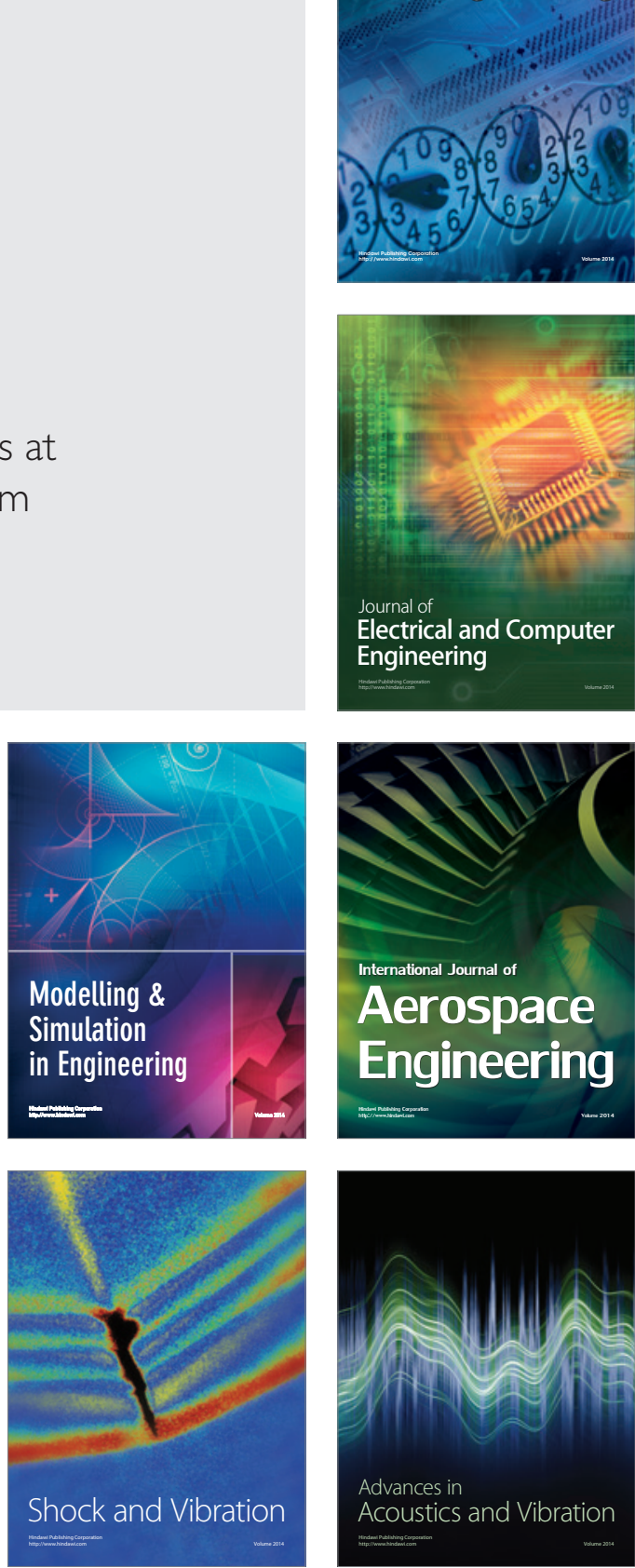